\title{
Functional Oligomers for the Control and Fixation of Spatial Organization in
}

\author{
Nanoparticle Assemblies (C.-K. Wu, K. Hultman, S. O’Brien, and J. T. Koberstein)
}

\section{Supporting Information}

Synthesis of $\gamma-\mathrm{Fe}_{2} \mathrm{O}_{3}$. The $\gamma-\mathrm{Fe}_{2} \mathrm{O}_{3}$ nanoparticles were synthesized using a method slightly modified from that of Hyeon et al. ${ }^{1}$ A solution of $3 \mathrm{~mL}(9.45 \mathrm{mmol})$ oleic acid and $15 \mathrm{~mL}$ (34.31 mmol) trioctylamine was heated to 320C under nitrogen atmosphere. When the temperature of the solution decreased to 200C, $0.4 \mathrm{~mL}(3.04 \mathrm{mmol})$ iron pentacarbonyl $\left(\mathrm{Fe}(\mathrm{CO})_{5}\right)$ (Sigma Aldrich) was injected and the solution was heated to 320C, and held for 1 hour. The color of the solution changed from its initial post injection color of orange to clear as the iron pentacarbonyl decomposed and then became black as $\mathrm{FeO}$ nanoparticles formed. After 1 hour, the solution was cooled to below $60 \mathrm{C}$ and $0.7 \mathrm{~g}$ (9.32 mmol) of trimethylamine-N-oxide was added. The solution was then held for 2 hours at $130 \mathrm{C}$ and $320 \mathrm{C}$ for 1 hour. The iron oxide solution changed from black to reddish-brown color and then back to black as the nanoparticles oxidized. The solution of $\gamma-\mathrm{Fe}_{2} \mathrm{O}_{3}$ was then cooled to below 40C and washed using chloroform and ethanol. Three diameters of monodisperse $\gamma-\mathrm{Fe}_{2} \mathrm{O}_{3}$ nanoparticles were used: 5.4, 9.5 and $13.5 \mathrm{~nm}$.

Monofunctional Carboxylic Acid Terminated PDMS (PDMS-COOH). Three different molecular weights $(M n=1000,5000$ and 10,000) of monofunctional carboxylic acid terminated polydimethylsiloxane (PDMS-COOH) were used as acquired from Polymer Source. The molecular weights determined by GPC are summarized in Supporting Table 1. The GPC (Shimazu) was calibrated with monodisperse polystyrene standards. The molecular weight and the polydispersity indices were calculated by applying a Q factor of 0.595 as reported by Jones. ${ }^{2}$ The structures of these PDMS-COOH are detailed in 
Supporting Figure 1. The density of PDMS of molecular weight $N_{P D M S}$ and with $\mathrm{COOH}$ end groups is ${ }^{3,4}$

$$
\rho_{P D M S}=\frac{1}{2}\left[\left(0.9673-44.9 / N_{P D M S}\right)+\left(0.9673+38.74 / N_{P D M S}\right)\right]
$$
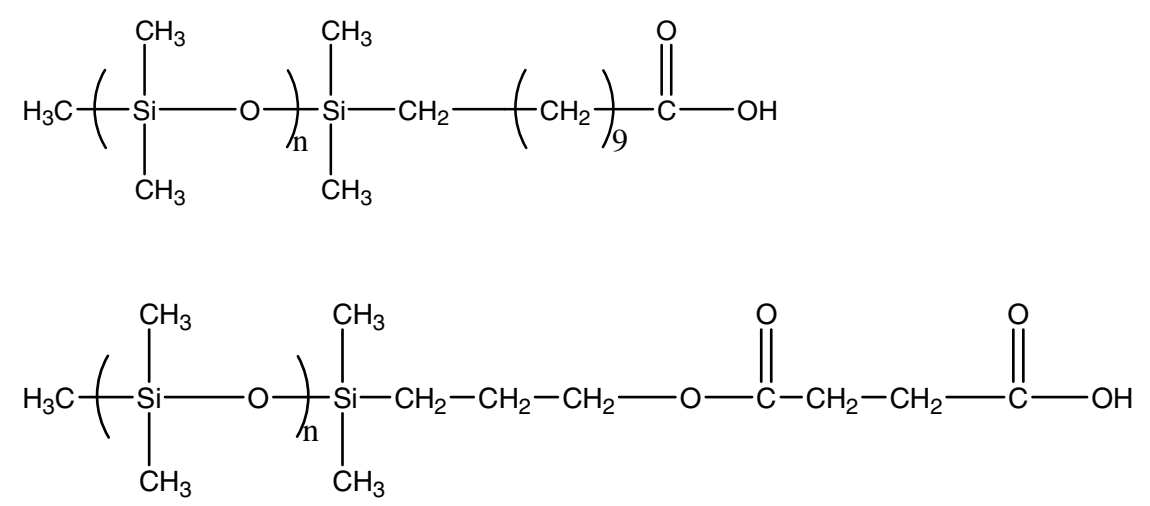

Supporting Figure 1. The chemical structures of the PDMS-COOH $(\mathrm{Mn}=1000)$, top, and PDMS-COOH (Mn=5000 and 10000), bottom.

Supporting Table 1. GPC characterization results for PDMS-COOH materials

\begin{tabular}{|c|c|c|c|c|}
\hline & $M n$ & $M w$ & $M n-$ GPC & $M w$-GPC \\
\hline & Supplied & Supplied & (as measured) & (as measured) \\
\hline PDMS-COOH1K & 1000 & 1300 & 959.1 (1612) & $1068(1795)$ \\
\hline PDMS-COOH5K & 5000 & 5700 & $5146(8649)$ & $5452(9163)$ \\
\hline PDMS-COOH10K & 10000 & 11000 & $10123(17014)$ & 11393 (19149) \\
\hline
\end{tabular}

Ligand Exchange. After synthesis, the $\gamma-\mathrm{Fe}_{2} \mathrm{O}_{3}$ nanoparticles were dispersible in chloroform by virtue of the oleic acid capping ligands. The oleic acid ligands were removed by sonicating a solution of the oleic acid coated $\gamma-\mathrm{Fe}_{2} \mathrm{O}_{3}$ nanoparticles, to which methanol was added, in a cooling bath for 6 hours. The bare $\gamma-\mathrm{Fe}_{2} \mathrm{O}_{3}$ nanoparticles were collected as a black precipitate after centrifugation and then vacuum dried. To introduce 
the polymeric ligands, the bare $\gamma-\mathrm{Fe}_{2} \mathrm{O}_{3}$ nanoparticles were mixed with PDMS-COOH in hexane and then sonicated for 8 hours in a cooling bath. Excess PDMS-COOH was removed by adding methanol followed by centrifugation. The PDMS-modified $\mathrm{Fe}_{2} \mathrm{O}_{3}$ nanoparticles were readily re-dispersed in hexane.

$\mathrm{Fe}_{2} \mathrm{O}_{3}$-PDMS Film Preparation. Films of PDMS-modified $\mathrm{Fe}_{2} \mathrm{O}_{3}$ nanoparticles were prepared by spin coating ( $2500 \mathrm{rpm} ; 1$ minute or by dip coating followed by slow solvent evaporation in a hexane rich chamber. Films were annealed in vacuum at $60 \mathrm{C}$ for 5 hours. Instrumentation. FTIR analysis (Nicolet 560) was used to confirm the ligand exchange reaction and the presence of direct chemical bonding between the nanoparticles and the ligands. The $\gamma-\mathrm{Fe}_{2} \mathrm{O}_{3}$ nanocrystallites and the PDMS-modified $\mathrm{Fe}_{2} \mathrm{O}_{3}$ nanoparticle films were characterized by TEM (JEOL CX100) with an accelerating voltage of $100 \mathrm{kV}$. Image analyses of the particle size, 2D FFT power spectra, interparticle spacing, and autocorrelation functions were carried out using Image $^{5}$ and Digital Micrograph software. ${ }^{6} \mathrm{X}$-ray diffraction patterns were obtained with an Inel X-ray diffractometer. The hydrodynamic radii of the PDMS-modified $\mathrm{Fe}_{2} \mathrm{O}_{3}$ nanoparticles were measured with a Brookhaven BI-200SM/BI900AT light scattering system. The ligand grafting density was measured by weight loss using a TA Q50 thermogravimetric analyzer. X-ray photoelectron spectroscopy spectra were recorded with a PHI 5500 spectrometer equipped with a hemispherical electron energy analyzer, a multichannel detector. The $\mathrm{Al}$ $\mathrm{K} \alpha$ monochromated X-ray source was run at $15 \mathrm{kV}$ and $23.3 \mathrm{~mA}$.

Confirmation of Ligand Exchange. Supporting Figure 2 confirms the success of the ligand exchange process as the infrared absorbance band characteristic of free carboxylic acid chain ends on the polymer is lost when the ligands attach to the nanoparticle surface. 
The FTIR spectra of the PDMS-modified $\mathrm{Fe}_{2} \mathrm{O}_{3}$ nanoparticle films $\left(\mathrm{d}=13.5 \mathrm{~nm}, M_{n}=1,000\right)$ document the disappearance of the peak at $1715 \mathrm{~cm}^{-1}$ seen in the free PDMS-COOH indicating that the carboxylic acid on the PDMS-COOH forms a direct bond with the $\gamma$ $\mathrm{Fe}_{2} \mathrm{O}_{3}$ nanoparticle surface and that there is no physisorbed PDMS on the nanoparticle surfaces. The bond between the acid group and the surface of $\gamma-\mathrm{Fe}_{2} \mathrm{O}_{3}$ nanoparticles has been found to be an organometallic covalent bond by Turro and co-workers. ${ }^{7}$ Analysis of the nanoparticles with $M_{n}=5,000$ and $M_{n}=10,000$ ligands is not possible because the low acid contents for these longer polymer chains are below the FTIR detection limit.

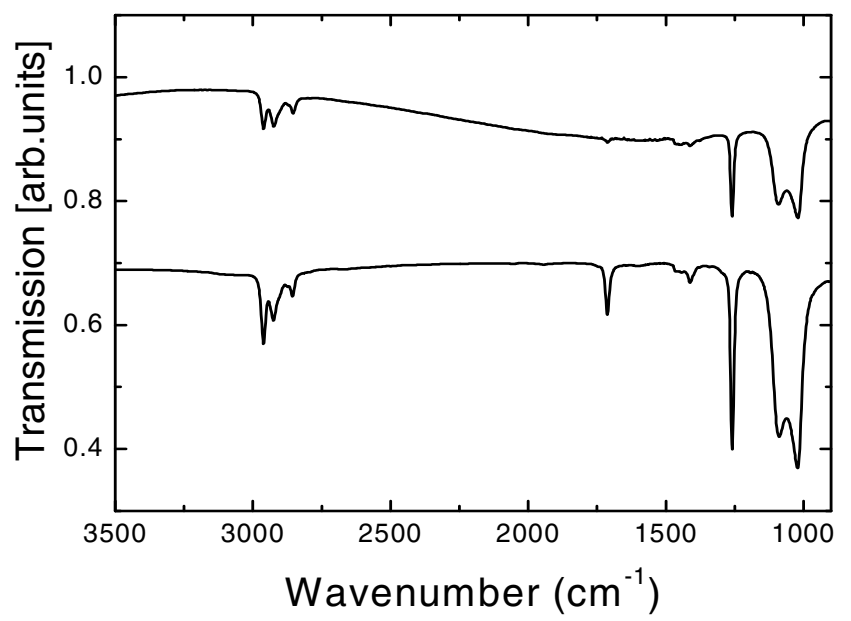

Supporting Figure 2. FTIR spectra of a PDMS-modified $\mathrm{Fe}_{2} \mathrm{O}_{3}$ nanoparticle film (d=13.5nm, $\left.M_{n}=1,000\right)$ (top), and PDMS-COOH $\left(M_{n}=1,000\right)$ (bottom).

The scattering results in Supporting Figure 3 illustrate how the ligand exchange process alters the individual nanoparticles. The hydrodynamic size of the ligand modified nanoparticles increases monotonically with length of the grafted PDMS ligands for all three nanoparticle sizes, providing evidence that the ligands tether to the nanoparticle by means of an interaction between the carboxylic end group and the oxide surface. Because 
the diameters obtained are of similar magnitude to those of individual nanoparticles, it can be concluded that ligand exchange provides for dispersion of individual nanoparticles and that aggregates are not present.

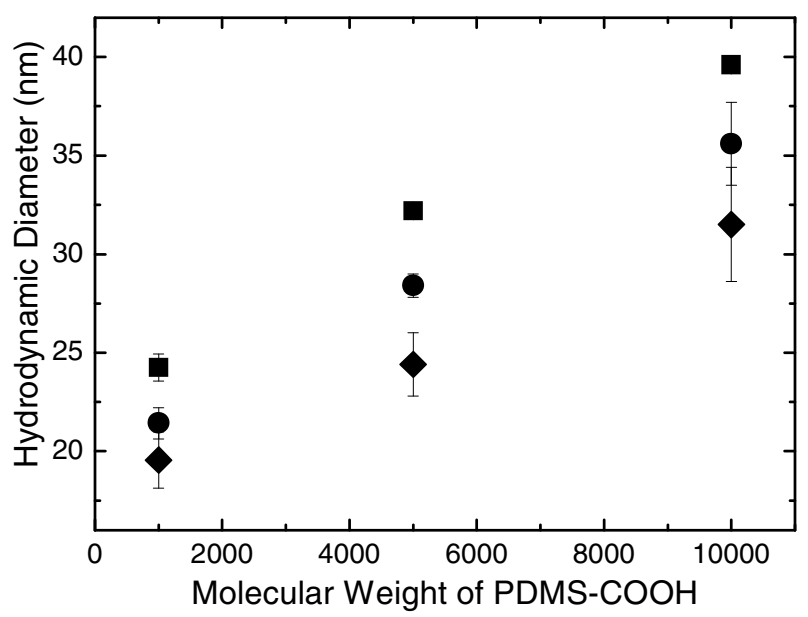

Supporting Figure 3. Hydrodynamic diameters of PDMS-modified $\mathrm{Fe}_{2} \mathrm{O}_{3}$ nanoparticles dispersed in hexane measured by dynamic light scattering (Nanoparticle diameters are: $5.4 \mathrm{~nm}$ (diamonds), $9.5 \mathrm{~nm}$ (circles) and $13.5 \mathrm{~nm}$ (squares).

Determination of the Grafting density $(\boldsymbol{\sigma})$. Determination of the polymer ligand surface density on nanoparticles is challenging for PDMS ligands. Normally, thermal gravimetric analysis, TGA, is applied to estimate the surface grafting density of polymers on nanoparticles by measuring weight loss after burning off carbonaceous ligands in an oxidative environment. Application of this method to characterize the oleic acid modified nanoparticles is shown in Supporting Figure 3. Unfortunately, this oxidative TGA method does not work for the PDMS ligands because they do not burn off but rather form silicon oxide upon oxidation. Because analysis of the converted silicon oxide is difficult, we are forced to estimate the ligand areal density by measuring the weight change of the hybrid 
nanoparticles in a reductive environment. The reductive environment removes the PDMS, however, the TGA analysis is complicated by the fact that iron oxide (i.e. maghemite) reduces gradually under an oxygen deficient environment at high temperature (i.e. > 400C) $)^{8,9}$ as shown in Supporting Figure 4 (b and c).

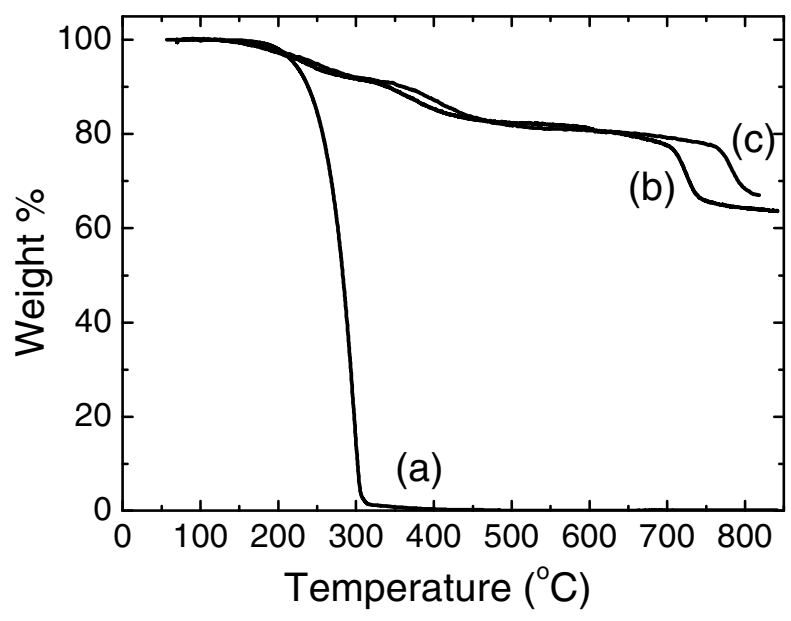

Supporting Figure 4. TGA curves of: (a) oleic acid (heating rate-20C/min) and oleic acid coated $9.5 \mathrm{~nm} \gamma-\mathrm{Fe}_{2} \mathrm{O}_{3}$ nanoparticles (b) $(20 \mathrm{C} / \mathrm{min}$ ) and (c) $80 \mathrm{C} / \mathrm{min}$. The weight loss observed in curves (b) and (c) above $400^{\circ} \mathrm{C}$ is due to the reduction of iron oxide.

The reduction of iron in the nitrogen atmosphere can be confirmed by comparing the TGA curves for oleic acid modified iron oxide nanoparticles under oxidative and reductive conditions shown in Supporting Figure 5. Curve 5(c) indicates that the oleic acid is removed fully at a temperature of about 300C under oxidative conditions. Under reductive conditions, curve 5(a), additional weight loss is observed above 300C due to reduction of the iron oxide nanoparticles. If the reductive environment is switched to oxygen at $850 \mathrm{C}$, as shown in curve $5(\mathrm{~b})$, the weight recovers to that of fully oxidized iron, represented by curve 5(c) at that same temperature. 


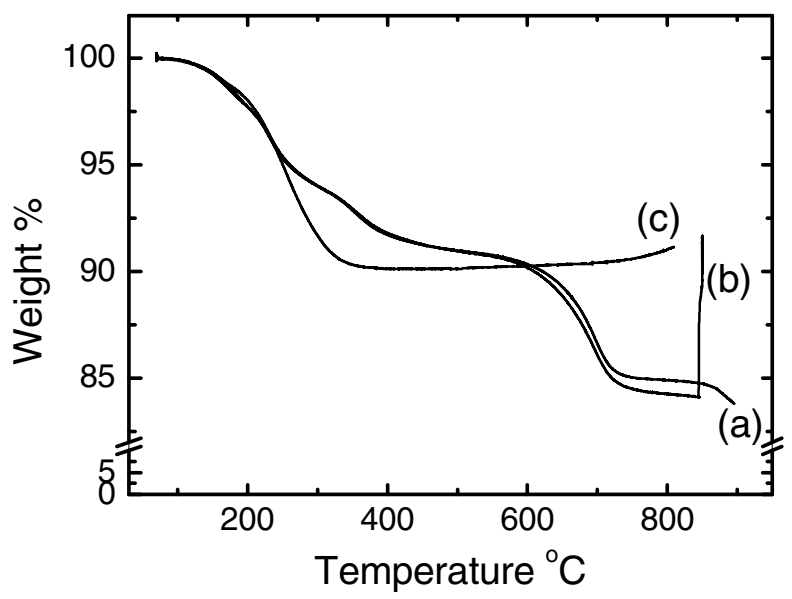

Supporting Figure 5. TGA data of oleic acid grafted $\gamma-\mathrm{Fe}_{2} \mathrm{O}_{3}(\mathrm{~d}=13.5 \mathrm{~nm})$ nanoparticles under nitrogen at heating rate of $10^{\circ} \mathrm{C} / \mathrm{min}(\mathrm{a})$, under oxygen at heating rate of $10^{\circ} \mathrm{C} / \mathrm{min}$ (c), and under nitrogen at heating rate of $10^{\circ} \mathrm{C} / \mathrm{min}$ till $850^{\circ} \mathrm{C}$ then switched to oxygen isothermally for 60 minutes (c).

TGA curves for PDMS-modified $\mathrm{Fe}_{2} \mathrm{O}_{3}$ nanoparticle films heated in a nitrogen environment are shown in Supporting Figure 6. It has been reported previously that the properties of polymers can be enhanced by incorporating nanoparticles into the polymer matrix. ${ }^{10}$ The thermal stability of the PDMS ligands is increased after complexation with the nanoparticle surface, as the TGA curves are all shifted to higher temperatures for endtethered PDMS. That is, PDMS bound to the nanoparticles decomposes at a higher temperature than does free PDMS.

Analysis of the weight loss curves must take into account the reduction of the iron oxide as the temperature is raised in a nitrogen environment. Three stages of reduction or weight loss are apparent in TGA curve (a) in Supporting Figure 4 at temperature intervals of $350-600 \mathrm{C}, 600-800 \mathrm{C}$, and above $800 \mathrm{C}$. We believe these three stages correspond to reduction of $\mathrm{Fe}_{2} \mathrm{O}_{3}$ to $\mathrm{Fe}_{3} \mathrm{O}_{4}, \mathrm{Fe}_{3} \mathrm{O}_{4}$ to $\mathrm{FeO}$ and $\mathrm{FeO}$ to $\mathrm{Fe}$, respectively. 

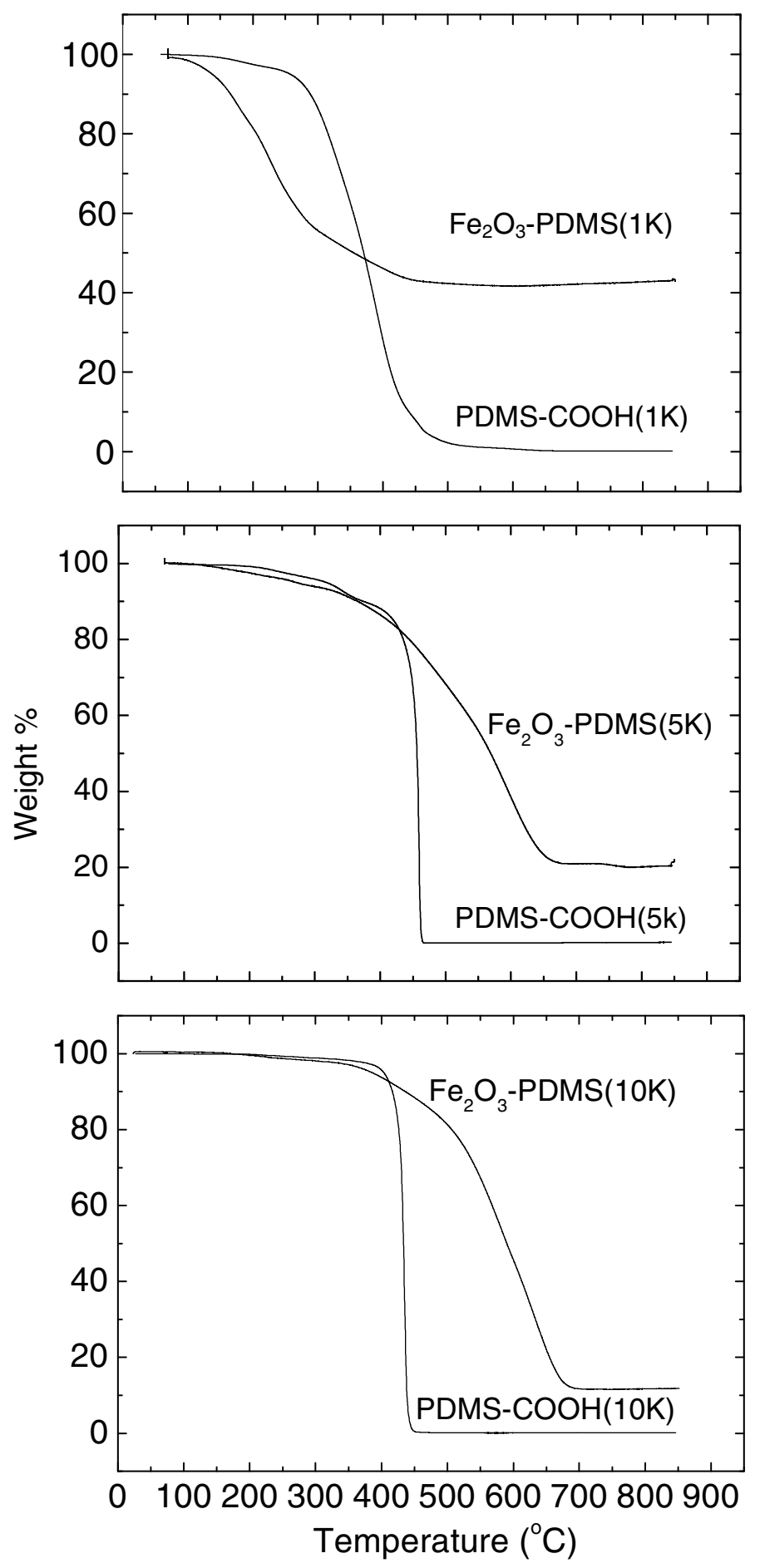

Supporting Figure 6. TGA curves of PDMS-modified $\mathrm{Fe}_{2} \mathrm{O}_{3}$ nanoparticle films $(\mathrm{d}=$ $13.5 \mathrm{~nm}$ ) and the free PDMS-COOH under nitrogen. Ligand molecular weights are 1000 (top), 5000 (middle), and 10000 (bottom). 
The grafting density may also be determined by analysis of the nanoparticle packing in the two dimensional films. The model adopted for this purpose is a volume filling hexagonal packing model with constant film thickness as shown in Supporting Figure 7. The volume of PDMS per nanoparticle can be determined from the measured values of $l$ (i.e. the lateral particle spacing) and $d$ (i.e. the diameter of the nanoparticles). A value for the grafting density, that is the number of ligands per nanoparticle, is calculated by dividing the total PDMS volume by the volume per ligand. For hexagonal packing within a continuous film of constant thickness $l$, the PDMS volume is

$$
V_{P D M S}=\frac{\sqrt{3}}{2} l^{2} \times l-\frac{4}{3} \pi\left(\frac{d}{2}\right)^{3}
$$

and the areal density of ligands is

$$
\sigma=\frac{\left[\left(V_{P D M S} \cdot \rho_{P D M S}\right) / N_{P D M S}\right] \cdot N_{A}}{4 \pi(d / 2)^{2}}
$$

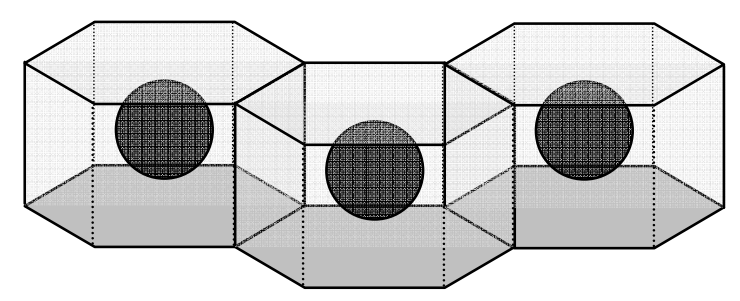

Supporting Figure 7. Schematic representation of the volume filling hexagonal packing model.

An important question to answer is how the ligands pack at the surface of the nanoparticle. The PDMS-COOH $(M n=1000)$ ligand is terminated with what is equivalent to a fatty acid of chain length of 11 . The close-packed area per molecule for stearic acid (with 18 carbons) adsorbed onto iron has been reported to be $.189 \mathrm{~nm}^{2} /$ molecule, ${ }^{11}$ corresponding to an areal density of 5.3 molecules $/ \mathrm{nm}^{2}$. The maximum surface densities 
reported for Langmuir-Blodgett (LB) films of a variety of fatty acids are of similar magnitude, falling in the range of 5-6 chains $/ \mathrm{nm} .{ }^{12}$ The experimental grafting density for the PDMS-COOH $(1 \mathrm{~K})$ is $4.0 \pm 1.3$ molecules $/ \mathrm{nm}^{2}$, indicating that the fatty-acid-like chain ends on the polymer can be accommodated in a nearly close-packed configuration at the surface. Does this organization, however, allow for packing of the PDMS backbone?

In studies of PDMS monolayers on water ${ }^{13}$ we found that the close-packed areas per molecule (for $M n=1000$ ) fell between .5 and $1.2 \mathrm{~nm}^{2}$ decreasing with increasing strength of interaction between the end group and water. This range corresponds to close-packed areal densities of 0.7-2 molecule/nm ${ }^{2}$. If we assume that the PDMS chain begins about 1 $\mathrm{nm}$ from the nanoparticle surface, estimated from ChemDraw, the ligand density for the PDMS backbone would be reduced by a factor of $\{(r+1 \mathrm{~nm}) / r\}^{2}$ or ca. 1.3, where $r$ is the nanoparticle radius. The highest areal density for the PDMS backbone is therefore estimated to be $3 \pm 1$ molecule $/ \mathrm{nm}^{2}$, close to the range of experimental close-packed areal densities. The highest density of packing for a PDMS chain occurs when it assumes the cis-trans configuration ${ }^{13}$, with a cross sectional area of $.52 \mathrm{~nm}^{2}$. The corresponding ligand areal for this configuration is 2.5 molecules $/ \mathrm{nm}^{2}$; comparable to the estimated areal density of the PDMS backbone. The results of the analysis suggest that the PDMS chain (i.e., for $M n=1000$ ) can also be accommodated as a nearly close-packed assembly where it meets the layer of fatty-acid-like chain ends. The PDMS ligands can adopt progressively less constrained configurations farther from the surface as the steric crowding decreases with distance from the surface. 
It is useful to compare the radius of gyration $\left(R_{g}\right)$ of free PDMS-COOH with the grafted PDMS-COOH brush height in order to confirm whether the PDMS ligands are configured as "brushes" on nanoparticle surface. Ignoring the functional end groups on PDMS-COOH, the $R_{g}$ for PDMS of polymerization index $N_{\mathrm{PDMS}}$ is ${ }^{14}$

$$
<R_{g}^{2}>=0.077 \times N_{P D M S}
$$

The simplest estimate of the experimental brush height, $h$, is one half of the distance between adjacent particle surfaces, or $h=\frac{l-d}{2}$. The results in Supporting Table 2 show that $R_{g}$ is smaller than $h$ for all three PDMS ligands, confirming that the PDMS ligands are brushes that stretch away from the nanoparticle surface.

Supporting Table 2. Radius of gyration and brush height for PDMS ligands

\begin{tabular}{|c|c|c|c|}
\hline $\begin{array}{l}\text { Molecular Weight } \\
\text { PDMS-COOH }\end{array}$ & $R_{g}(\mathrm{~nm})$ & $\begin{array}{c}h[\mathrm{~nm}] \\
(d=13.5 \mathrm{~nm})\end{array}$ & $\begin{array}{c}h[\mathrm{~nm}] \\
(d=9.5 \mathrm{~nm})\end{array}$ \\
\hline 1000 & 1.0 & 3.0 & 3.1 \\
\hline 5000 & 2.0 & 5.8 & 5.0 \\
\hline 10000 & 2.8 & 8.0 & 7.6 \\
\hline
\end{tabular}

Conversion of PDMS to SiOx by UV/Ozone treatment. The PDMS ligands are transformed to silicon oxide ( $\mathrm{SiOx}$ ) by a UV/ozone process. The conversion is monitored by x-ray photoelectron spectroscopy (XPS) as shown in Supporting Table 3. The O/Si atomic ratio for PDMS-COOH and PDMS-modified $\mathrm{Fe}_{2} \mathrm{O}_{3}$ nanoparticles is about 1 , as expected from the chemical structure of PDMS. The O/Si ratio should reach a value of 2.0 after conversion because $\mathrm{Si}-\mathrm{CH}_{3}$ groups in $\mathrm{PDMS}$ are replaced by $\mathrm{Si}-\mathrm{O}$; however the ratio reaches a value of 2.5. The origin of this apparent discrepancy is that an $\mathrm{O}_{1 \mathrm{~s}}$ signal associated with the $\gamma-\mathrm{Fe}_{2} \mathrm{O}_{3}$ nanoparticles is observed after UV/ozone conversion, as 
shown in Supporting Figure 8. The $\mathrm{O}_{1 \mathrm{~s}}$ peaks from PDMS and $\mathrm{SiO}_{\mathrm{x}}$ appear at $532.5 \mathrm{eV}$ while the $\mathrm{O}_{1 \mathrm{~s}}$ peak on $\mathrm{Fe}_{2} \mathrm{O}_{3}$ is seen at $530 \mathrm{eV}$. The high resolution $\mathrm{Si}_{2 \mathrm{p}}$ spectra in Supporting Figure 9 suggest complete conversion to $\mathrm{SiOx}$ as the binding energy for PDMS-COOH shifts from $101.5 \mathrm{eV}$ to $102.6 \mathrm{eV}$ after the UV/ozone treatment, consistent with a transformation from two to four oxygen atoms in the tetrahedral coordination shell. The probe depth of XPS is only of the order of 10nm, however, so we can only state conclusively that the top 10nm or so of PDMS is converted to SiOx.

Supporting Table 3. Atomic concentrations from XPS analyses

\begin{tabular}{|c|c|c|c|c|}
\hline \multirow{2}{*}{ Material } & \multicolumn{4}{|c|}{ Atom } \\
\cline { 2 - 5 } & $\mathrm{C}$ & $\mathrm{O}$ & $\mathrm{Si}$ & $\mathrm{Fe}$ \\
\hline PDMS-COOH & $50.2 \%$ & $25.0 \%$ & $24.8 \%$ & $0 \%$ \\
\hline $\mathrm{Fe}_{2} \mathrm{O}_{3}$-PDMS & $51.9 \%$ & $25.1 \%$ & $23.0 \%$ & $<0.1 \%$ \\
\hline $\mathrm{Fe}_{2} \mathrm{O}_{3}$-SiOx & $10.9 \%$ & $62.4 \%$ & $25.1 \%$ & $1.6 \%$ \\
\hline
\end{tabular}

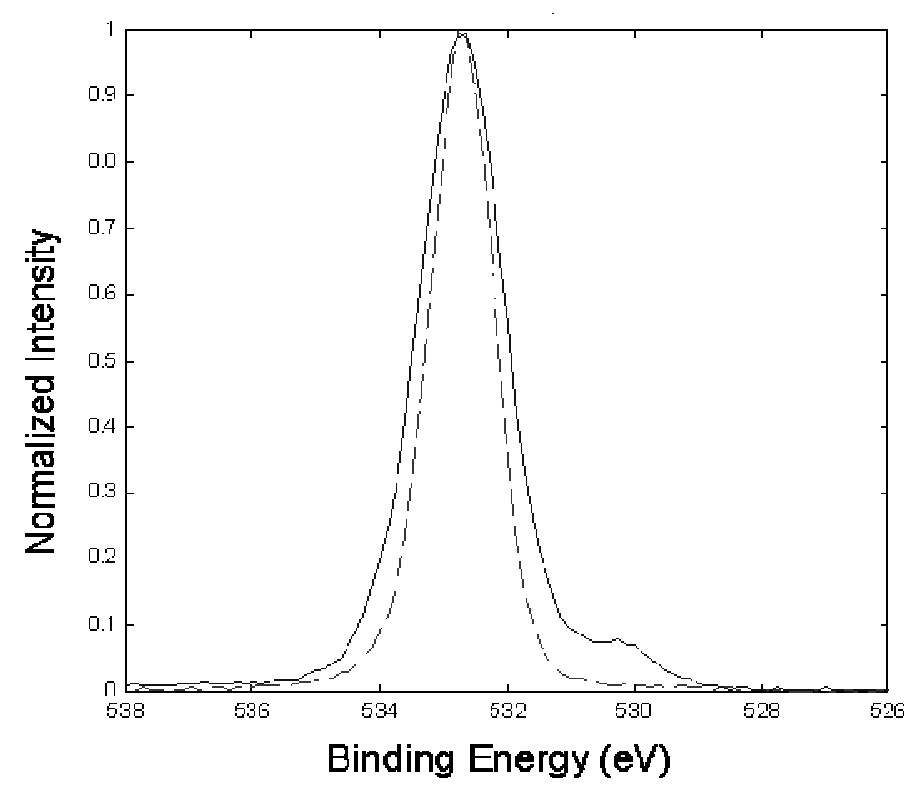

Supporting Figure 8. The $\mathrm{O}_{1 \mathrm{~s}}$ XPS spectra of a PDMS-modified $\mathrm{Fe}_{2} \mathrm{O}_{3}$ film before UV/ozone treatment (dashed line), and after UV/ozone treatment for 60 minutes (solid line). 


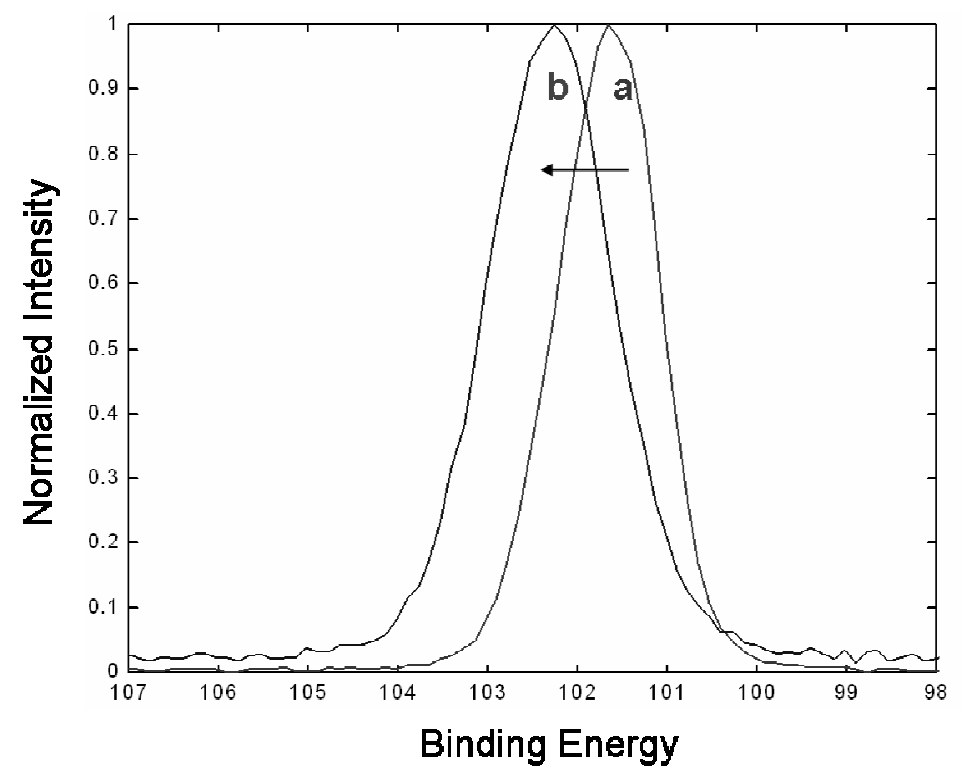

Supporting Figure 9. High resolution $\mathrm{Si}_{2 \mathrm{p}}$ XPS spectra of a PDMS-modified $\mathrm{Fe}_{2} \mathrm{O}_{3}$ film before (a), and after (b) the UV/ozone conversion.

2D FFT Power Spectrum. The procedure for determining the lateral particle spacing is shown schematically in Supporting Figure 10. The TEM image is first converted to a binary black-and-white image by thresholding. The binary image is then fast Fourier transformed to get the power spectrum. A radially averaged profile plot is taken on the power spectrum to derive the averaged power spectrum intensity versus frequency. The first peak on the plot, which contains the information of separation, is fitted with Gaussian to derive a more accurate $\Delta k$ value. The lateral particle spacing, $l$, (i.e. the distance between the centers of two particles) is derived from the FFT satellite peak separation from center, $\Delta k \cdot{ }^{15}$ For hexagonal packing the spacing is $l=1 /\left(\Delta k \cdot \sin 60^{\circ}\right) .{ }^{16,17}$ 


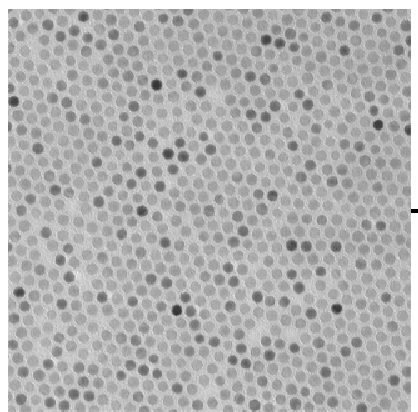

TEM Image

Filter, Threshold
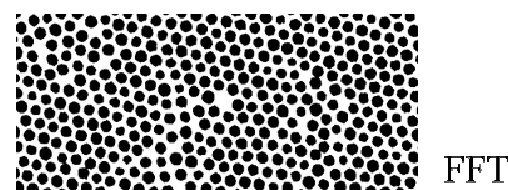

Binary Image
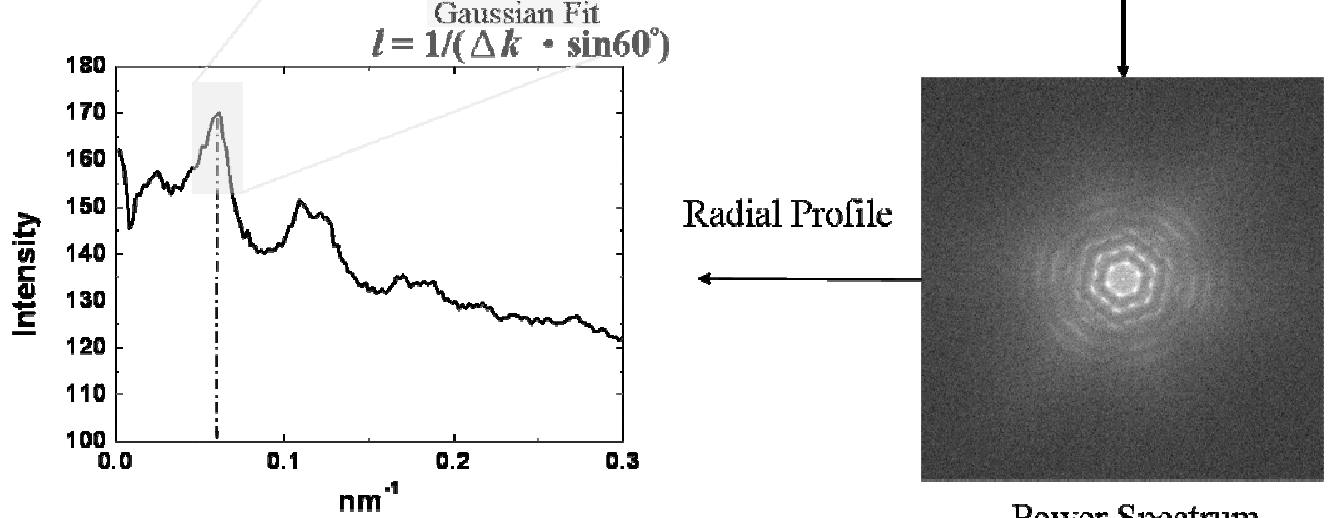

Power Spectrum

Supporting Figure 10. The procedure for 2D FFT image analysis of nanoparticle arrays.

The lateral particle spacing $(l)$ can be derived from the power spectrum.

Autocorrelation Functions. The regularity/order in each $\mathrm{Fe}_{2} \mathrm{O}_{3}-\mathrm{PDMS}$ nanoparticle array is quantified by analysis of the autocorrelation function $C(r)$. A lateral correlation length, $\xi$, describing the persistence of order in the $\mathrm{Fe}_{2} \mathrm{O}_{3}$ nanoparticle arrays is determined by fitting an exponential decay function, $C(r) \sim \exp (-r / \xi)$ to the experimental autocorrelation functions. ${ }^{15}$ The procedure for calculating the autocorrelation by analysis of the TEM images is schematically summarized in Supporting Figure 11. 


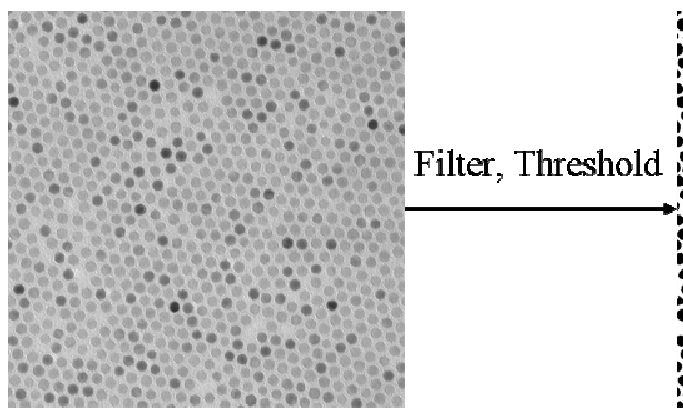

TEM Image

Binary Image
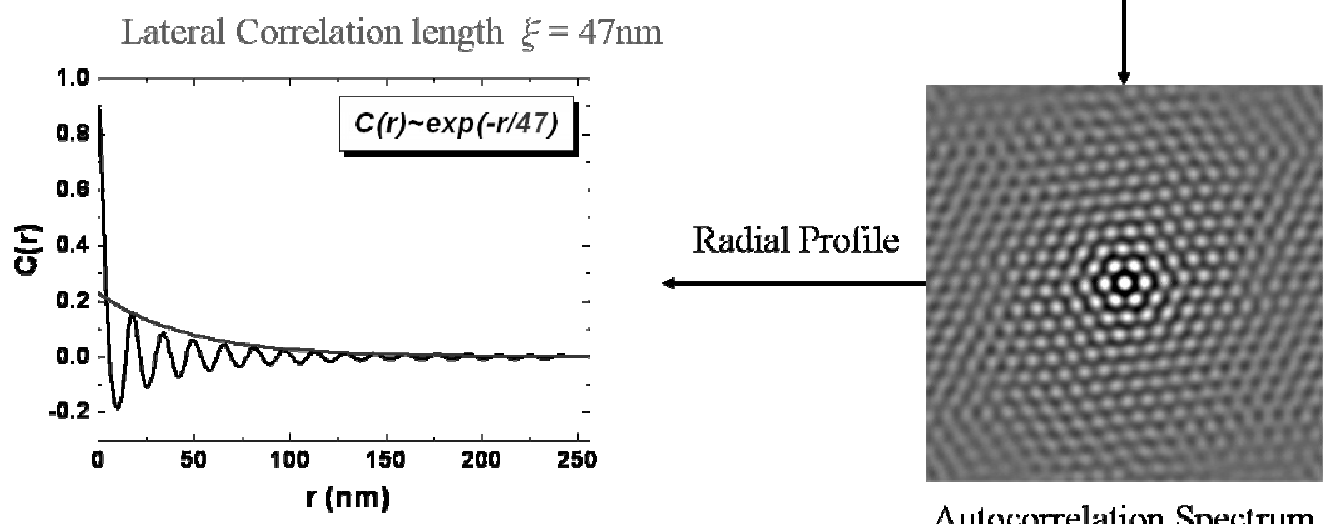

Autocorrelation Spectrum

Supporting Figure 11. The procedure employed to determine the lateral correlation length

$(\xi)$ from the autocorrelation function of the nanoparticle arrays.

Supporting References

1. Hyeon, T., Lee; S. S., Park, J.; Chung, Y.; Na, H. B. J. Am. Chem. Soc. 2001, 123, 12798.

2. Jones, F. R. Euro. Polym. J. 1974, 10, 249.

3. Jalbert, C.; Koberstein, J. T.; Hariharan, A.; Kumar, S. K. Macromolecules 1997, 30,4481 .

4. Jalbert, C. A. PhD Thesis, University of Connecticut, 1993.

5. http://rsb.info.nih.gov/ij/

6. http://www.gatan.com/imaging/dig_micrograph.html

7. Turro, N. J.; Lakshminarasimhan, P. H.; Jockusch, S.; O'Brien, S.; Grancharov, S. 
G.; Redl, F. X. Nano Lett. 2002, 2, 325.

8. Darken, L. S.; Gurry, R. W. J. Am. Chem. Soc. 1945, 67, 1398.

9. Darken, L. S.; Gurry, R. W. J. Am. Chem. Soc. 1946, 68, 798.

10. Laachachi, A.; Leroy, E.; Cochez, M.; Ferriol, M.; Lopez Cuesta, J. M. Poly. Degrad. Stab. 2005, 89, 344.

11. Timivions, C. O.; Patterson, R. L.; Lockhart, L.B., Jr. J. Coll. Interface Sci. 1968, $26,120$.

12. Roberts, G. Langmuir-Blodgett Films. (Plenum Press: New York and London, 1990).

13. Lenk, T. L.; Lee, D. H. T.; Koberstein, J. T. Langmuir 1994, 10, 1857.

14. Zilliox, J. G.; Roovers, J. E. L.; Bywater, S. Macromolecules 1975, 8, 573.

15. Teichert, C. Phys. Rep. Rev. Sect. Phys. Lett. 2002, 365, 335.

16. Raab, A.; Lechner, R. T.; Springholz, G. Appl. Phys. Lett. 2002, 80, 1273.

17. Pinczolits, M.; Springholz, G.; Baucer, G. hys. Rev. B 1999, 60, 11524. 